\title{
Bessere Versorgungsstrukturen für Menschen mit seltenen Krankheiten
}

\author{
Hermann Amstad ${ }^{a}$, Agnes Nienhaus ${ }^{b}$ \\ ${ }^{a}$ Dr. med., Generalsekretär SAMW, Bern; ${ }^{b}$ lic. phil., Geschäftsführerin unimedsuisse, Bern
}

Heute sind weltweit rund 6000 bis 8000 seltene Krankheiten bekannt. Gemäss internationaler Übereinkunft wird eine «Seltene Krankheit» definiert als eine Krankheit, die

- in weniger als 5 Fällen pro 10000 Einwohnerinnen und Einwohner auftritt sowie

- lebensbedrohlich oder chronisch invalidisierend ist. In der Schweiz sind diverse Organisationen entstanden, die sich für die Belange von Menschen mit seltenen Krankheiten einsetzen - auch auf politischer Ebene. Der Bundesrat hat deshalb das Bundesamt für Gesundheit (BAG) beauftragt, in Zusammenarbeit mit den betroffenen Organisationen und Fachpersonen und mit den Kantonen ein «Nationales Konzept Seltene Krankheiten» zu erarbeiten. Dieses Konzept wurde im Herbst 2014 veröffentlicht; es formuliert 7 Ziele und sieht 19 Massnahmen vor, um diese Ziele zu erreichen.

\section{Nationales Konzept schlägt «Referenzzentren» vor}

Mit dem Nationalen Konzept Seltene Krankheiten sollen die vielfältigen Herausforderungen bei der Behandlung und Betreuung von Patientinnen und Patienten mit seltenen Krankheiten gelöst werden. Namentlich an die Errichtung von Referenzzentren sind grosse Erwartungen geknüpft. Das Nationale Konzept Seltene Krankheiten hält dazu Folgendes fest: "Um die Diagnose innert nützlicher Frist zu stellen, die Qualität der Versorgung zu verbessern, die Weiterbildung und Forschung zu unterstützen, werden Referenzzentren errichtet. Das Ziel dieser Zentren ist es, die Patienten, deren Angehörige und Gesundheitsfachleute fachlich kompetent zu unterstützen und die Kenntnisse zu bündeln. Notwendig ist eine entsprechende Zusammenarbeit zwischen den verschiedenen Stellen, um insbesondere die Koordination der Behandlung zu verbessern. Die Qualifikation als Referenzzentrum bedingt die Erfüllung [spezifischer] Kriterien [...]. Dazu soll ein entsprechender Prozess definiert werden.»

Das Nationale Konzept schlägt Referenzzentren mit einem breiten Aufgabenspektrum als Lösungsansatz für die Erfüllung sämtlicher bestehender Bedürfnisse vor. Untersuchungen zu den effektiven Lücken in der Versorgung, Koordination, Forschung etc. fehlen allerdings für die Schweiz. Es ist deshalb unklar, ob für alle Anliegen Referenzzentren die besten Lösungen darstellen bzw. ob es sinnvolle alternative Lösungsansätze gäbe. Internationale Vergleiche zeigen, dass Referenzzentren ganz unterschiedlich konzipiert und umgesetzt werden; abhängig vom jeweiligen Gesundheitssystem des Landes und von den Finanzierungsmöglichkeiten.

Das BAG gelangte im Februar 2015 mit der Anfrage an die SAMW, den Prozess zur Errichtung von Referenzzentren zu definieren (Massnahme 1, Nationales Konzept). Da keine gesetzliche Grundlage bestehe, solle ein «Gentlemen's Agreement» angestrebt werden. Die erste Fassung eines entsprechenden Berichts stiess im Herbst 2015 in der Vernehmlassung auf teilweise heftige Kritik. Der Bericht wurde in der Folge umfassend überarbeitet und Ende Juni 2016 dem BAG überreicht.

\section{Grundsätze}

Der Bericht geht davon aus, dass sich die Umsetzung von Massnahme 1 an folgenden Grundsätzen orientieren sollte:

- Lösungen werden auf jener Ebene gesucht, die dafür am besten geeignet ist (Subsidiaritätsprinzip); das heisst, die verschiedenen Anliegen müssen nicht alle von einer einzigen Struktur abgedeckt werden.

- Patientennähe - «Médecine de proximité» - in der Versorgung: Die Versorgung muss von den Patientenwegen her gedacht werden. Bestehende Versorgungsstrukturen und Angebote sollen möglichst erhalten bleiben.

- Referenzzentren für Krankheitsgruppen sind in der Mehrzahl der Fälle ungeeignet. Die Entwicklung der Medizin bedingt vorab einen systemischen Ansatz. Ähnlich wie eine Krebserkrankung nicht primär organspezifisch, sondern systemisch - in einer Klinik für Onkologie - behandelt wird, braucht es auch für Patienten mit seltenen Krankheiten eher krankheitsübergreifende Kompetenzzentren, die mit den spezifischen Problemen und Bedürfnissen dieser Patientengruppe vertraut sind. 
Tabelle 1: Versorgungsnetzwerke aus spezialisierten Leistungserbringern und (in definierten Fällen) Referenzzentren.

\begin{tabular}{|c|c|}
\hline \multirow[t]{2}{*}{ Ziele } & $\begin{array}{l}\text { Die Patientinnen und Patienten erhalten wohnortnah qualitativ hoch- } \\
\text { stehende Behandlung, Betreuung und psychosoziale Unterstützung. }\end{array}$ \\
\hline & Die Netzwerke organisieren sich selbst. \\
\hline \multirow[t]{7}{*}{ Aufgaben } & $\begin{array}{l}\text { Die Fachspezialisten und ihre Netzwerke sind für folgende Aufgaben } \\
\text { zuständig: }\end{array}$ \\
\hline & - Versorgung \\
\hline & - Forschung, Aus- und Weiterbildung \\
\hline & $\begin{array}{l}\text { - Erarbeitung von krankheitsspezifischen Behandlungspfaden } \\
\text { und Guidelines in Absprache mit den Plattformen für seltene } \\
\text { Krankheiten }\end{array}$ \\
\hline & - Teilnahme an krankheitsspezifischen (internationalen) Registern \\
\hline & - Teilnahme an internationalen Referenznetzwerken \\
\hline & - Kooperation mit Organisationen der Selbsthilfe \\
\hline \multirow[t]{4}{*}{ Organisation } & $\begin{array}{l}\text { Die Netzwerke organisieren sich selbst und verteilen innerhalb } \\
\text { ihrer Mitglieder die unterschiedlichen Rollen (u.a. Netzwerklead, } \\
\text { internationale Vertretung). }\end{array}$ \\
\hline & $\begin{array}{l}\text { Die Netzwerke sind krankheitsspezifisch organisiert (nach Möglich- } \\
\text { keit nach grösseren Krankheitsgruppen) und umfassen Leistungs- } \\
\text { erbringer auf dem Sekundärniveau (Fachärzte und Zentrumsspitäler) } \\
\text { und dem Tertiärniveau (Universitätsspitäler). }\end{array}$ \\
\hline & $\begin{array}{l}\text { Innerhalb der Netzwerke nehmen die Universitätsspitäler eine } \\
\text { besondere Rolle ein, indem sie für die Forschung, Aus- und } \\
\text { Weiterbildung sowie für die Registerführung verantwortlich } \\
\text { zeichnen. }\end{array}$ \\
\hline & $\begin{array}{l}\text { Ist die Schaffung eines Referenzzentrums für eine spezifische } \\
\text { Krankheit oder Krankheitsgruppe sinnvoll, wird dies im Netzwerk } \\
\text { abgestimmt und in der Nationalen Koordination beschlossen. }\end{array}$ \\
\hline \multirow[t]{2}{*}{ Anforderungen } & $\begin{array}{l}\text { Versorgungsnetzwerke: Antrag durch die Netzwerkbeteiligten und } \\
\text { die betroffene/n Patientenorganisation/en }\end{array}$ \\
\hline & $\begin{array}{l}\text { Referenzzentren: Erfüllung vorgegebener Kriterien } \\
\text { (vgl. SAMW-Bericht «Referenzzentren» 2016, Anhang I) }\end{array}$ \\
\hline
\end{tabular}

Tabelle 2: Plattformen für Seltene Krankheiten.

\begin{tabular}{|c|c|}
\hline \multirow[t]{2}{*}{ Ziele } & $\begin{array}{l}\text { Patienten und Leistungserbringer verfügen mit den Interdisziplinä- } \\
\text { ren Plattformen für seltene Krankheiten über Anlaufstellen bei } \\
\text { unklarer Diagnose und für «vernachlässigte» seltene Krankheiten } \\
\text { (vgl. Empfehlungen der SAMW aus dem Jahr 2014). }\end{array}$ \\
\hline & $\begin{array}{l}\text { Sicherung/Vereinfachung der Finanzierung der Leistungen bei } \\
\text { seltenen Krankheiten }\end{array}$ \\
\hline \multirow[t]{4}{*}{ Aufgaben } & Die Plattformen sind für folgende Aufgaben zuständig: \\
\hline & $\begin{array}{l}\text { - Anlaufstellen für die Abklärung von Patienten mit komplexen } \\
\text { seltenen Krankheiten oder mit Verdacht auf seltene Krankheiten; }\end{array}$ \\
\hline & - Care Management / Behandlungskoordination \\
\hline & $\begin{array}{l}\text { - Beschreibung von krankheitsübergreifenden Versorgungsprozes- } \\
\text { sen und Beratungsangeboten in Absprache mit den anderen } \\
\text { Plattformen, Netzwerken und Patientenorganisationen }\end{array}$ \\
\hline \multirow[t]{3}{*}{ Organisation } & $\begin{array}{l}\text { Ca. } 5 \text { bis } 6 \text { Plattformen - im Idealfall je } 1 \text { Plattform pro Universitäts- } \\
\text { spital bzw. pro universitären Standort und } 1 \text { Plattform im Tessin }\end{array}$ \\
\hline & $\begin{array}{l}\text { Die Anlaufstellen funktionieren interdisziplinär und krankheitsüber- } \\
\text { greifend. }\end{array}$ \\
\hline & $\begin{array}{l}\text { Die Plattformen sollen keine ausgedehnten Organisations- } \\
\text { einheiten darstellen, verfügen jedoch in der Regel über eigenes } \\
\text { Fachpersonal. }\end{array}$ \\
\hline Anforderungen & $\begin{array}{l}\text { Erfüllung vorgegebener Kriterien (vgl. SAMW-Bericht «Referenz- } \\
\text { zentren» 2016, Anhang II) }\end{array}$ \\
\hline Trägerschaft & $\begin{array}{l}\text { Universitätsspitäler und forschungskompetente grosse Kantons- } \\
\text { spitäler }\end{array}$ \\
\hline
\end{tabular}

- Eigentliche «Referenzzentren» für bestimmte seltene Krankheiten sind in Fällen vorzusehen, in denen

a. die Abklärung und Behandlung einer seltenen Krankheit besonders anspruchsvoll ist,

b. die entsprechende Expertise in einem Schweizer Spital vorhanden ist, und/oder

c. die internationale Vernetzung in einem «Netzwerk von Referenzzentren» angezeigt ist.

- Falls in der Schweiz für eine bestimmte Krankheit keine adäquaten Behandlungsangebote bestehen, soll die Behandlung in einem ausländischen Zentrum möglich sein.

- In Europa wird die Netzwerkbildung für seltene Krankheiten vorangetrieben und so das vorhandene Fachwissen zu seltenen Krankheiten geteilt, für Patientinnen und Patienten einfacher zugänglich gemacht (im Hinblick auf die Versorgung) und für die Forschung erschlossen. Der Anschluss an diese internationalen Entwicklungen ist auch für die Schweiz wichtig.

\section{Organisationsstrukturen: Netzwerke, Referenzzentren, Plattformen}

Der SAMW-Bericht schlägt vor, die im Konzept genannten Anliegen auf zwei Ebenen umzusetzen:

Die Basis besteht aus krankheits(gruppen)spezifischen Versorgungsnetzwerken der Leistungserbringer (Fachärzte und Spitäler, siehe Tabelle 1). In klar definierten Fällen und sofern die Kriterien erfüllt sind, können innerhalb solcher Netzwerke für bestimmte seltene Krankheiten Referenzzentren bezeichnet werden. Es gilt die Regel: Wo die Versorgung gut funktioniert, bleibt sie auf dieser ersten Ebene angesiedelt.

Falls das Angebot auf der ersten Ebene nicht genügt bzw. Lücken bestehen, können die Patienten auf einer zweiten Ebene «Plattformen für Seltene Krankheiten» aufsuchen (siehe Tabelle 2). Diese fungieren als interdisziplinäre Anlaufstellen zur Abklärung und Koordination von Behandlungen, erfüllen Informationsaufgaben und übernehmen die Koordination von Fachpersonen. Die Bildung von Versorgungsnetzwerken und Referenzzentren erfolgt bottom-up durch die involvierten Leistungserbringer in Zusammenarbeit mit den Patientenorganisationen; sie kann nicht vorgeschrieben, sondern höchstens unterstützt werden. Die Schaffung von Plattformen für Seltene Krankheiten liegt in der Verantwortung der Universitätsspitäler und - soweit sinnvoll der grossen Kantonsspitäler. Die vorgeschlagenen Strukturen der Versorgungsnetzwerke, der Referenzzentren und der Plattformen müssen gewisse Anforderungen erfüllen (siehe Tabelle 3) und bedürfen der Anerkennung durch ein übergeordnetes Gremium. 


\section{Aufbau- und Bezeichnungsprozess}

Korrespondenz:

Dr. Hermann Amstad

Haus der Akademien

CH-3001 Bern

h.amstad[at]samw.ch Generalsekretär SAMW

Der bisherige Prozess hat verdeutlicht, was das BAG mit dem Auftrag kommunizierte: Für den Aufbau neuer Versorgungsstrukturen für Patienten mit seltenen Krankheiten ist ein "Gentlemen's Agreement» notwendig; er kann nicht top-down angeordnet bzw. umgesetzt

Tabelle 3: Versorgungsstrukturen für Seltene Krankheiten.

\begin{tabular}{|c|c|c|}
\hline & Anforderungen & Anerkennung durch \\
\hline $\begin{array}{l}\text { Versorgungsnetzwerk } \\
\text { für eine bestimmte seltene } \\
\text { Krankheit(sgruppe) }\end{array}$ & $\begin{array}{l}\text { Antrag durch die Netzwerk- } \\
\text { beteiligten und die betroffene } \\
\text { Patientenorganisation }\end{array}$ & $\begin{array}{l}\text { Nationale Koordination } \\
\text { für Seltene Krankheiten }\end{array}$ \\
\hline $\begin{array}{l}\text { Referenzzentrum für } \\
\text { eine bestimmte seltene } \\
\text { Krankheit }\end{array}$ & $\begin{array}{l}\text { Erfüllung der Kriterien ge- } \\
\text { mäss Anhang I des Berichts } \\
\text { und } \\
\text { Antrag durch den Leistungs- } \\
\text { erbringer, Unterstützung durch } \\
\text { die zuständige Patienten- } \\
\text { organisation }\end{array}$ & $\begin{array}{l}\text { Nationale Koordination } \\
\text { für Seltene Krankheiten }\end{array}$ \\
\hline $\begin{array}{l}\text { Plattformen für seltene } \\
\text { Krankheiten }\end{array}$ & $\begin{array}{l}\text { Erfüllung der Kriterien ge- } \\
\text { mäss Anhang II des Berichts }\end{array}$ & $\begin{array}{l}\text { Nationale Koordination } \\
\text { für Seltene Krankheiten }\end{array}$ \\
\hline
\end{tabular}

Tabelle 4: Nationale Koordination Seltene Krankheiten.

\begin{tabular}{|c|c|}
\hline \multirow[t]{3}{*}{ Ziele } & $\begin{array}{l}\text { Selbstorganisation von Leistungserbringern, Betroffenen und } \\
\text { anderen Stakeholdern zugunsten einvernehmlicher Lösungen }\end{array}$ \\
\hline & $\begin{array}{l}\text { Die Behörden verfügen über eine Ansprechstelle im Bereich } \\
\text { "Seltene Krankheiten». }\end{array}$ \\
\hline & $\begin{array}{l}\text { Die internationale Anbindung der Schweiz im Bereich "Seltene } \\
\text { Krankheiten» ist sichergestellt. }\end{array}$ \\
\hline \multirow[t]{6}{*}{ Aufgaben } & Die nationale Koordination übernimmt folgende Aufgaben \\
\hline & $\begin{array}{l}\text { - Abstimmung der Aufgaben und Rollen auf nationaler und } \\
\text { internationaler Ebene. }\end{array}$ \\
\hline & $\begin{array}{l}\text { - Koordination der Plattformen, Versorgungsnetzwerke und Referenz- } \\
\text { zentren; Gestaltung und Durchführung der Anerkennungsprozesse }\end{array}$ \\
\hline & $\begin{array}{l}\text { - Bezeichnung der Schweizer Vertreter in den internationalen } \\
\text { Referenznetzwerken für seltene Krankheiten. }\end{array}$ \\
\hline & - Einbezug Patientensicht auf übergeordneter Ebene \\
\hline & - Kommunikation mit Verwaltungsstellen (Bund, GDK) \\
\hline Organisation & $\begin{array}{l}\text { Verein mit einer Geschäftsstelle (angehängt am Sitz der Univer- } \\
\text { sitären Medizin Schweiz). }\end{array}$ \\
\hline Trägerschaft & $\begin{array}{l}\text { Universitätsspitäler und Spitäler mit Forschungskompetenz, } \\
\text { SAMW, ProRaris }\end{array}$ \\
\hline Partner & $\begin{array}{l}\mathrm{H}+\text {, Interpharma, Krankenversicherer (Dachverbände), BAG, GDK } \\
(\rightarrow \text { Advisory Board) }\end{array}$ \\
\hline Finanzierung & $\begin{array}{l}\text { Mitgliederbeiträge der Trägerorganisationen, ev. Beiträge Bund und } \\
\text { Kantone }\end{array}$ \\
\hline
\end{tabular}

werden. Der Zusammenschluss von Leistungserbringern zu krankheits(gruppen)spezifischen Netzwerken, in Zusammenarbeit mit den zuständigen Patientenorganisationen, muss von der Basis angestossen und realisiert werden. Es liegt in der Verantwortung der grossen Zentrumsspitäler, in Kooperation krankheitsübergreifende «Plattformen für Seltene Krankheiten» zu schaffen.

Dieser Aufbauprozess wird eine gewisse Zeit in Anspruch nehmen, zumal derzeit keine finanziellen Anreize vorgesehen sind. Gleichzeitig bedarf es einer Steuerung und Koordination, idealerweise auch einer Unterstützung durch ein übergeordnetes Gremium. Last, but not least darf die Qualitätssicherung nicht ausser Acht gelassen werden. Diese Aufgaben - namentlich auch die Anerkennung von Versorgungsnetzwerken, Referenzzentren und Plattformen für seltene Krankheiten - können von einer "Nationalen Koordination Seltene Krankheiten" übernommen werden (siehe Tabelle 4). Diese Nationale Koordination soll von den beteiligten Akteuren gemeinsam getragen werden und als übergeordnete Trägerschaft dafür sorgen, dass einvernehmliche Lösungen gefunden werden und ein «Gentlemen's Agreement» erreicht werden kann.

\section{Ausblick}

Menschen mit seltenen Krankheiten sind mit zahlreichen Einschränkungen und Herausforderungen konfrontiert; verständlicherweise erhoffen sie sich vom Nationalen Konzept Seltene Krankheiten möglichst rasch eine Verbesserung ihrer Situation. In einem föderalistisch organisierten Gesundheitssystem wie der Schweiz können jedoch die notwendigen Veränderungen nicht einfach top-down verordnet werden. Vielmehr ist ein sorgfältig geplanter Aushandlungsprozess nötig - und dieser braucht Zeit. Nachdem der SAMWBericht im Juni 2016 dem BAG übergeben wurde und Anfang November das Kickoff-Meeting für die geplante Nationale Koordination Seltene Krankheiten stattfand, ist dieser Prozess in Gang.

Die vollständige Fassung des SAMW-Berichts ist auf der Website zu finden: samw.ch/empfehlungen. 